\title{
INDEX
}

Aceh, Sultanate of, and War (1873-1904) 90, 95-8, 101-2, 231

Adowa (battle, 1896) 207, 209, 215-16, 219-22

advertising 74-5

Africa 13, 62, 78

Africa, southern 1, 70, 74, 79

Afrikaner nationalism 114-15, 116, 117, 119

Afrikaners 231

see also Great Trek

Agence de la France d'outre-mer 45

Agence économique des colonies 43

Agence générale des colonies 36

Albert I (King of the Belgians) 124, 133-4, 139

Algeria 23-5, 28, 30, 35-7, 39, 46, 126 see also French conquest of Algeria

Algerian War of Independence (1954-62) 45,151

Algérianiste literary movement 33

Alldeutscher Verband (Pan-German League) 168,175

Alsace-Lorraine 230

American Revolutionary War (1775-83) $59,65,66$

Amery, Leopold 11

anthropology 7, 168

anti-imperialism 167

antisemitism 184, 187

Antwerp 129, 131, 135, 136, 137, 143, 144

Arcade du Cinquantenaire (Liège, 1905) 132

Asia 62, 78

Association Internationale Africaine 127, 169

Auslandsdeutsche (Germans abroad) 166, 167,232

Aussiedler (greater Germany) 232

Austen, Jane 69

Australia 12, 60, 63, 69, 70, 78

Austrian Succession War (1740-48) 59, 65

Axum obelisk (Rome, 1938) 220
Baden-Powell, Sir Robert 79, 181

Ballantyne, R.M. 74

Banania (brand) 34, 37, 39

bandes dessinées see comics, comic books, comic strips

Bara, Max 148

Bastien, Alfred 134, 142

Battaglia, Roberto 198, 208

Baudouin (King of the Belgians) 125, 150

Bauer, Ludwig 124

Bayly, C.A. 232

Belgian Congo 1, 2, 8, 9, 10, 124-52

Belgische Werkliedenpartij see Parti Ouvrier Belge

Belgium 10, 12, 14, 124-52

Belzoni, Giovanni Battista 66

Berger, Stefan 232

Berlin conference (1884-85) 127

Bertrand, Louis 33

Bevione, Giuseppe 213

Birdwood, Sir George 76

Bismarck, Otto von 10, 165

Bloch, Marc 230

Bloembergen, M. 100, 110, 119

Bodelschwingh, Friedrich von 174

Boe, Gérard de 146

Boissevain, Charles 102, 109

book market (Italy) 204-5

Bossenbroek, Martin 91, 94, 101-2, 108, 109

Bosworth, Richard 217-18

Botanical Gardens, Berlin 169

botany 169

Bouccicoult, Dion 66

Bougainville, Louis-Antoine de 20

Brandt, Henry 146

Brazil 172

Brazza, Savorgnan de 28, 29, 32

Bremen, Colonial Monument 183

see also monuments and memorials

Britain see United Kingdom

British empire 6, 10, 11, 13, 57-82

Britishness 197-9

broadsheet ballads 204-7, 218

Brunialti, Attilio 203 
Brussels 124, 130, 132, 136, 139, 143, 151

Buchan, John 71

Caine, Hall 66

Cameroon 172, 181

Camus, Albert 41, 44, 46

Canada 60, 63, 68, 69, 71, 78

Cape Colony 60

Caribbean 13, 60, 78

Casement, Roger 131

cash crops 171

Catholic Party (Belgium) 133

Catholicism 127, 199, 202, 211-12, 219

Catholics 129

Cattier, Félicien 132

Cauvin, André 146

Cavell, Edith 139

Centre d'Accueil pour le Personnel Africain (Belgium) 150

Césaire, Aimé 47

Chabod, Federico 203

Chamberlain, Joseph 4

Charles X (King of France) 23

China 2, 12, 78

Christian missions see missions, missionaries

Church Missionary Society 174

Churchill, Winston 230

Ciarlo, David 172

cinema 5, 113, 130, 134-5, 138, 145-6, 179-80, 218-19

Cinéma Colonial (Belgium) 134-5

citizenship 167-8, 197-8, 200, 203, 213, 221,224

Citröen missions (Croisière jaune and Croisière noire) 38

civilising mission 28

class (social) 197-8, 200-2, 204, 221, 224

Clemenceau, Georges 27

coffee 172

Collins, Wilkie 69

colonial fantasies 163,184

Colonial Institute (the Netherlands) $111-12$

'Colonial lobby' (Belgium) 129, 130

Colonial party (France) 20, 36

colonial veterans $136,138-9,148,151$, 152

Comaroff, John and Jean 1, 173 comics, comic books, comic strips 131, $137-8,148$

commemorative services 209-12

Commission of Enquiry on Congo atrocities (Belgium, 1905) 132

commodities 169-73

commodity racism 171

communists 151

conférenciers (Belgium) 145, 146

Congo 124-52

see also Belgian Congo

Congo Free State see État Indépendant du Congo

Congo Reform Association 131

Congolese 125, 129, 130, 131, 135, 138, 142-3, 144, 148, 149-50, 151

Congorama (light and video show) 149

Conseil économique (France) 137

Conseil supérieur des colonies (France) 137

consensus for national state (Italy) 196, $199,217,221,223$

consumption imperialism (of tropical colonial products, Germany) 171-3

Cook, Captain James 80

Corradini, Enrico 211, 214-16, 221

cotton 158-60

Couperus, Louis 100

Crespel, Louis 141

Crispi, Francesco 199-201, 210-13, 217, $222,224-5$

Croce, Benedetto 210-12, 216

Cudell, Charles 128

D'Annunzio, Gabriele 216

Daudet, Alphonse 30

Day of Wedding Rings (Italy 1935) 220-1

Debertry, Léon 145

Del Boca, Angelo 198-9, 214, 216

Delacroix, Eugène 24, 25

Delagoa Bay 105

Delmer, Louis 131

Dernburg, Bernhard 185

Deutsche Flottenverein (DFV) 175-81

Deutsche Kolonialgesellschaft (DKG) 139, 166, 175, 176-80, 182, 183

Dhanis, Francis 135

Diagne, Blaise 129

Dickens, Charles 69, 70

Dinet, Etienne 30, 39

Dogali (battle, 1887) 204, 206-9 


\section{N DEX}

Dorgelès, Roland 40

Douwes Dekker, E. (Multatuli) 95

Doyle, Sir Arthur Conan 71

Duggan, Christopher 199

Durham Report 61

Dutch East Indies 126, 127

Dutch empire 10, 11, 90-123

Dutch South Africa Association (NZAV) 104, 105, 107

Duveyrier, Henri 25

Eberhardt, Isabelle 33

Eboué, Félix 43

Ecole coloniale (France) 31

Ecole d'Alger (France) 40-1

École mondiale (Belgium) 131

economic imperialism 170

Edinburgh 229

education and schooling 1, 128, 131, 137, 141, 144-5, 195-7, 199, 216, 219

Egypt, French expedition to (1798) 21

Eley, Geoff 175

Elgar, Sir Edward 77

Elizabeth II (Queen of Great Britain) 63

emigration 61-4, 128, 143, 145, 165-7

see also German emigrant diaspora

emigration problem (Italy) 201, 214, 217-18, 223

Empire Marketing Board (United Kingdom) 75, 137

Eritrea 201-2, 210, 223

État Indépendant du Congo 124, 126, $127,128,129,131,132,137,140$, $141,145,150,151,171,231$

'ethical policy' (ethische politiek, the Netherlands) 92, 98, 99, 110, 111

Ethiopia 195, 200, 204, 206, 208-9, 211 , 217-23

Etienne, Eugène 27, 36

Exhibitions 8, 14, 20, 25, 28, 31, 37-8, $75-7,100-1,129,130,132,134$, $136,142,143,148-50,151,181-2$

exploration 164

expositions universelles see Exhibitions

Faidherbe, Louis 24

Fanon, Frantz 47

Fasci Siciliani 201

Fascism 195-7, 217-23

Fascists 232

Fashoda crisis (1898) 27, 31
Felice, Renzo de 219-20

Ferry, Jules 27

film see cinema

First World War (1914-18) 12, 13, 34, 67, $110,112,129,136,137,139,143$, $144,162,165,170,185,202,204$, 217

Fischer, Eugen 168

Fitzpatrick, Matthew 163

Flaubert, Gustave 25

Flemings (Belgium) 127, 143

Force publique (Belgium) 128, 142

Foucauld, Charles de 30, 41, 42, 44

Fourth Republic (France) 232

France 10, 12, 14, 19-48, 71, 126, 128, $129,143,151$

Franchetti, Leopoldo 200-3, 217

franchise 1

Free French 232

French conquest of Algeria (1830-47) 23-4, 26

French empire 10, 11, 13

French Foreign Legion 164

French Union (post-1945) 46

Frenssen, Gustav 179

Frère-Orban, Walthère 127

Frobenius, Leo 168

Fromentin, Eugène 25

Fuhrmann, Wolfgang 180

Gaffarel, Paul 28

Gallieni, Joseph 32

Gandhi, Mohandas 129

Gauguin, Paul 30

Gautier, Théophile 25

Geiser, Jean 30

gender 176-7

see also women

genocide 184

Genval, Ernest 138

geographical societies (France) 26-7

geography 169

German Colonial Department 158

German East 184, 185 see also Ostkolonisation

German East Africa 167, 172, 173, 180

German emigrant diaspora 166, 169

German empire 10, 12, 158-87

German Navy 165, 176, 178, 180

German South-West Africa (Namibia) $126,167,181,185$ 
German Weltpolitik 161

Germany 4, 10, 12, 14, 71, 126, 128, $136,139,140,144,158-87,231$, 232

Gesellschaft für deutsche Kolonisation 128

Ghent (Belgium) 134, 142, 150

Gibraltar, Siege of (1779-83) 66

Gillain, Joseph 138

Giolitti, Giovanni 212-17, 222-3

Girardet, Raoul 20

Girault, Charles 132

Gladstone, William Ewart 10

globalisation 161, 170

Glorie, Ingrid 114

Gordon, General Charles 66, 71, 80

Gouda, Frances 99

Gourdinne, Ernest 138

Graaff, Bart de 113

Graaff, Bob de 109, 111, 113

Great Britain see United Kingdom

Great Depression (1929-34) 14

Great Trek 103, 106, 115

see also Afrikaners

greater Britain 232

greater Germany 232

greater Netherlands 232

Grosse, Pascal 168

Guatemala 172

guerre scolaire 128

Hagenbeck, Carl 181-2

Haggard, Sir Henry Rider 71

Hal (Belgium) 150

Hall, Catherine 78

Hasselt (Belgium) 150

Hassert, Kurt 170

Henry de la Lindi, Josué 140

Henty, G.A. 74

Hergé (Georges Remi) 37, 138

heroes 6, 61

Hertzka, Theodor 185

Herzl, Theodor 185

Heutsz, J.B. van 96-7, 98, 100

Heyman, André 146

Highland Clearances 62

Hildebrand, Klaus 183

Hitler, Adolf 221

Hobhouse, Emily 71

Hobson, J.A. 57-8, 67

Hochschild, Adam 126
Holocaust 184

Huigen, Siegfried 106

Hungry Forties 62

hunting and wildlife 179

Imperial Order Daughters of the Empire 60

India 58, 65, 68, 69, 72, 126

indigenous peoples 9

Indochina 24, 27, 30, 33, 40, 45-6

see also Khmer art

industry and industrialisation 127, 135, $136,138,142$

'informal imperialism' 2, 3

Institut colonial (France) 36

Ireland 62, 63, 70

Italian empire 10, 195-225

Italy 4, 10, 13, 14, 128, 195-225

Italy, southern 195, 200-2, 216

Ixelles (Belgium) 141

Janssen, Frans 140, 141, 143

Japan 7, 12, 15, 145

Java 94-5, 96, 100, 102, 113

Jijé see Gillain, Joseph

jingoism 204, 206, 213-14, 216, 220

Joris, Frans 135

Journées Coloniales (colonial pressure group, Belgium) 141, 145, 147

journées coloniales /colonial commemorations, Belgium) 141, 147

juvenile literature (the Netherlands) 102, $112,115,118,119$

Kaye, M.M. 72

Kenya 126

Khmer art 24

Kimbanguism 137

Kipling, Rudyard 70, 72

Kitawala movement 145, 150

Koch, Robert 169

koloniale dagen see journées coloniales

Koloniale Reichsarbeitsgemeinschaft 139

Koninklijke Museum voor Midden Afrika see Musée du Congo

Koppen, Chris 105

Kröll, Ulrich 107

Kruger, Paul 105, 107, 108, 115

Kuitenbrouwer, Maarten 93, 99

Kulturkampf 173

Kundrus, Birthe 184 


\section{N DEX}

\section{L'Illustration 27}

La Pérouse, Comte de 20

Labanca, Nicola 208-9

Lanaro, Silvio 203

Latin America 2, 15

Laude, Norbert 144

Lavigerie, Charles Martial 28

Le Petit Journal 27, 36

Le Petit Parisien 27, 36

Le Temps 27, 36

Le Tour du Monde 24, 27

League of Nations 139

Lebensraum 169

Leblond, Marius 40

Legêne, Susan 94-5

Légion étrangère see French Foreign Legion

Leloir, Léon 137-8

Lemaire, Charles 137, 144

Leopold II (King of the Belgians) 8, 10, $124-33,137,139,141-2,148$, $150-2$

Leopoldville 134, 150

Leroy-Beaulieu, Paul 28

Lesseps, Ferdinand de 25

Lettow-Vorbeck, Paul von 139

Levi, Carlo 195-6, 200, 204, 219, 224-5

Leyds, W.J. 107, 114, 115

Liberalism (Italy) 196, 198, 202-3, 211-12, 221-3

Liberals (Belgium) 129

Libya 208, 210, 213-17, 223, 225

Libyan war (1911-12) 216

Liège 132

literacy 199-200, 204, 216

literature 58, 68, 131, 137, 147-8, 149 see also juvenile literature (the Netherlands)

Livingstone, David 77, 78, 80

Lombok 96, 97, 102, 232

London 131, 144

Loti, Pierre 30

Low, D.A. 1

lus sanguinis 168

Lyautey, Hubert 32, 34, 37, 40, 44

MacKenzie, John 20, 198, 221

Madagascar 126

magazines (Germany) 163-4

Mahan, Alfred Thayer 10

Malraux, André 40, 47
Mangin, Charles 34

Maran, René 40

Marchand, Jean-Baptiste 32-3

Martineau, Alfred 31, 37

Massola 129

Matadi 134

Mathieu, Paul 134, 142

Maupassant, Guy de 30

Max, Adolf 142-3

May, Karl 178-9

Memmi, Albert 47

Meredith, Hal 71

Midgley, Clare 60

Mignemi, Adolfo 218

military glory (Italy) 203, 206-7, 208, 210

Milner, Alfred 114

missions, missionaries $5,7,8-9,58$, 60-1, 68, 78-9, 128, 132, 136, 141, $142,145-6,173-5$

Mitterrand, François 45

monuments and memorials 131,135 , $136,138-9,140-1,142,145$, $147-8,150,151,152$

see also Bremen, Colonial Monument

Morel, E.D. 124, 131

Morris, William 76

Mouvement géographique 128

Musée du Congo (Tervuren, Belgium) or Musée Royal de l'Afrique Centrale $130,131,132-4,135-6,137,140$, $141-2,143,148$

museums 130-7, 140-3, 148, 170

music 77-8

Mussolini, Benito 196, 204, 214, 217, 219-24

Namur (Belgium) 137, 150

Napoleon I, Bonaparte (Emperor of the French) 65

Napoleonic Wars 60

Nationalverein 165

Nazi Germany 168

Nazi propaganda 230

Nelson, Admiral Horatio 65, 80

Netherlands, the 10, 12, 90-123, 126, 127,143

New Guinea 172

New Zealand 12, 60, 63, 69, 70, 78

Nieuwenhuys, Rob 100

Nigeria, Northern 8 


\section{INDEX}

Nora, Pierre 20

Office Colonial (Belgium) 136, 139, 140, 141,143

Olbrechts, Frans 149-50

Omissi, Dominic 72

Onafhankelijke Congostaat see État Indépendant du Congo

Optique Belge 130

Ostkolonisation 185

see also German East

Ostmarkenverein (German Association for the Eastern Provinces) 175-6

Ottoman empire 13

Pan-German League 168, 175

Parliament (Italian) 199, 212, 217

parti colonial (Belgium) 230

Parti Ouvrier Belge 128

Parti Socialiste Révolutionnaire 143

Pascoli, Giovanni 214-16, 223

Pattynama, Pamela 100

peasantry (Italian) 195-6, 201-2, 213-17, 224-5

Pennaroli (publisher) 204-7, 218

Penning, L. 115, 119

Périer, Gaston-Denys 142

Perino (Italian publisher) 207-8

Pétain, Marshal Philippe 43

Petit, Gabrielle 139

Philippines 127

photography 179

Piccinini, Giuseppe 208

Pinero, Arthur Wing 68

plumage trade 172

Plus Grande France, la 232

Poirier, Léon 42

political ecology 171

popular culture 177-82

Popular Front (France) 232

popular song (Italy) 196, 206, 216, 218

Porter, Bernard 197-8, 200, 213, 224

Portugal 126

Portuguese empire 8, 15-16, 60

press 58,62

Primrose League 128

'Proletarian Nation' (Italy) 210-11, 214

Putumayo 172

quinzaines coloniales (colonial fortnights, Belgium) 139, 141 race $166,168,177,184$

racism 61,81

radio $112-13,137$

Randau, Robert 33, 40

Reeves, Nicholas 230

Renkin, Jules 133-4

reprise (take-over of Congo by Belgium, 1908) 125, 132, 134

Rhodes, Cecil 63, 66, 74, 79

Risorgimento 199, 201, 208, 215-16

Rodogno, Davide 222

Rohmer, Sax 71

Rome 195, 201-3, 219-20, 224

Royal Dutch Geographical Society

(KNAG) 95-6

Royal Museum for Central Africa see Musée du Congo

Royal Netherlands Inst. of S.E. Asian and Caribbean Studies (KITLV) 99

Royale Union Coloniale (Belgium) 146

Ruanda-Urundi 139, 140

rubber 171

Rüger, Jan 180

Ruggio, Gian Luigi 215

Ruskin, John 76

Russian empire 6

Ryckmans, Pierre 148, 151

Sahara 25, 33, 38, 46

Saharomania 41, 44

Said, Edward 198

Saint-Exupéry, Antoine de 38, 41

Saint-Simonians 24

Salisbury, Robert 3rd Marquess of 10

Samoa 167

Sartre, Jean-Paul 47

Sas, Niek van 92, 119

Scandinavia 125, 128

Scheutists 128

Schillings, Carl Georg 179

Schirren, Hélène 151

school textbooks 216-17, 220, 223-4

Schreiner, Olive 72

Schumpeter, Joseph 231

Schutte, Gerrit 105

Schweitzer, Albert 169

Scotland 62

Scouting for Boys 181

see also Baden-Powell, Sir Robert

Scramble for Africa 15-16 


\section{IN DEX}

Second World War (1939-45) 12, 13, 42-4, 62, 113, 116-17, 143-4, 145, 184,223

Senegalese tirailleurs 34, 37

Senghor, Léopold Sédar 47, 168

Seven Years' War (1756-63) 59, 65

Short, John Phillip 178

Sicily 200-1, 216

Sielmann, Heinz 146

Simplicissimus 158-9

slavery 60

Smuts, Jan 108

Snouk Hurgronje, C. 98, 99

social Darwinism 4, 68, 76, 98

social imperialism 4-5, 160

'social question' (Belgium) 128

socialism 92, 102, 105, 110, 199, 204, 211-16, 219

socialists $129,149,151$

Société Belge d'Études et d'Expansion 146

Société d'histoire des colonies françaises 31

Société française pour l'abolition de l'esclavage 24

Société Royale Belge de Géographie 129

Somalia 223

Sonnino, Sydney 200

South Africa 231

South African War (1899-1902) 60, 71, $80,90,107-9,113,115,118,231$

Spain 232

Spanish-American War (1898) 10

Spanish empire 15

St Giles cathedral, Edinburgh 229

Stamverwantschap (Dutch-Afrikaner racial kinship) 93, 104

Stanley, Henry Morton 127, 128, 164

Stanley in Africa (play) 164

Steel, Flora Annie 72

Stengers, Jean 127, 150

Stern, Fritz 230

Stevenson, Robert Louis 71

students (German) 186-7

suffrage (Italy) 199, 202, 212, 215-16

Taselaar, Arjan 110, 111, 112, 113

telegraph cables 94, 107, 112

Terhoeven, Petra 221

Tervuren 126, 130, 131, 132, 135, 136, $137,140,141-2,143,148,150,151$

textbooks 125, 137, 139, 145, 147, 148
Thackeray, William 69

Tharaud, Jérôme and Jean 40

theatre $5,64-8,131,216$

Third Republic (France) 43, 232

Thompson, E.P. 2

Thorne, Susan 79

Thys, Albert 129, 130

Tintin 138

Tipu Sultan (of Mysore) 65

Tocqueville, Alexis de 24

totalitarianism 217-18, 222

Trans-Saharan railway (Transsaharien) 28

Transvaal 90, 103, 105-6, 107, 114, 115

Trollope, Anthony 63, 69, 70

tropical medicine 168-9

Turati, Filippo 214, 216

Twain, Mark 145

Übersiedler (greater Germany) 232

Uganda 8

Union Coloniale Française 128

United Kingdom 57-82, 128, 129, 134, $137,139,141$

universal expositions see Exhibitions

Université Coloniale (Belgium) 137, 144

USA $3,7,10,15,60,63-4,71,145,165$, 181

Van Iseghem, André 140

Vanthemsche, Guy 126

Veer, Pieter van der 98

Velde, Henk te 105, 109

Velde, Paul van der 95, 96

Vernet, Horace 25

Vernon, Admiral Edward 65, 80

Versailles, Treaty of 139

Verviers (Belgium) 138

veterinary science 169

Veth, P.J. 95-6, 98

Viaene, Vincent 126

Vichy France and the empire 43-4, 232

Victor Emmanuele II (King of Italy) 203, 210

Victor Emmanuele III (King of Italy) 219

Victoria (Queen of Great Britain) 61, 68, 77,232

Victoria League 60

Vietnam 126

see also Indochina

Vieuchange, Michel 41 
villages indigènes 130, 149, 150 see also Völkerschauen

villages nègres see villages indigènes

Vincart, L. 135-6

Vleeschauwer, Albert de 144

Vloo, Roger de 145

Völkerschauen ('shows of peoples', Germany) 181-2, 186, 231

Volksgemeinschaft (global panGermanism) 166

Walloons 127, 143

Warburg, Otto 185

Watchtower movement see Kitawala movement

Wauters, Jules 124, 128

Wehler, Hans-Ulrich 160, 170, 186

Weimar Republic 139, 167

wereldtentoonstellingen see Exhibitions

whiteness 167

Wiart, Carton de 142-3
Wilhelmina (Queen of the Netherlands) $92,93,98,107,111,113$

Wilson, Kathleen 59, 64

Winton, Francis de 128

Wolfe, General James 80

women 9, 60, 72, 79, 176-7 see also gender

World's Fairs see Exhibitions

Yonge, Charlotte M. 78

youth movements $79-80$

Zantop, Susanne 163

Zanzibar 141

Zimmerman, Andrew 168

Zionism 185

Zoographe (film pavilion, 1897, Belgium) 130, 135

Zoological Gardens, Berlin 182 